\title{
Dynamics of the formation of an event horizon
}

\author{
A. A. Shatskiy, A. Yu. Andreev \\ (Submitted 17 November 1988) \\ Zh. Éksp. Teor. Fiz. 116, 353-368 (August 1999)
}

\begin{abstract}
The radial motion of matter in a centrally symmetric gravitational field in a comoving reference frame is investigated for a realistic equation of state of matter. The dynamics of the formation of an event horizon are investigated.
\end{abstract}

\section{INTRODUCTION}

The formation of a black-hole event horizon has attracted a great deal of attention on the part of physicists for a long time. An enormous amount of material has been written on this subject (see, for example, Ref. [1, 2, 3, 4]); nevertheless, the treatment of this problem within the general theory of relativity has created more questions than known solutions.

One of the main questions concerning this problem is still the reciprocal influence of accreting matter on a black hole. The motion of test particles in the field of a black hole has been considered hitherto for the most part, but they, as we know, do not exhibit a reciprocal influence, which can be enormous when a falling particle achieves the speed of light as it crosses the event horizon.

1. In this paper we consider the special, but physically real case of spherically symmetric accretion on a central body without allowance for rotation. The following notation is adopted: the speed of light $c$ and the gravitational constant $G$ are set equal to unity. In these units the gravitational radius for a given mass $M$ is $r_{g}=2 M$, i.e., the radius of the event horizon in free space for the same mass concentrated at the center.

Let us devise a likely model for the evolution of the system. We assume that our system is a cooling massive star having a radius $R_{0}$ and a gravitational radius $R_{G_{0}}=2 M\left(R_{0}\right)$, where $R_{G_{0}}<R_{0}$. The matter comprising this body is initially at rest ("dust" with the equation of state $P=\alpha \varepsilon$, where $P$ is the pressure in the matter, $\varepsilon$ is the energy density, and $\alpha$ is a constant). In the next moment the matter begins to fall freely. [1] ] If it is assumed that the gravitational fields are not excessively strong and that the dust density [2] ] is fairly small in the initial moment, a force field with a finite energy is needed to retain it in the initial moment. After this field is removed, the dust leaves the system and ceases to interact with it after a time of the order of the size of the system, i.e., after a time much shorter than the 
time during which the dust manages to partially settle and the gravitational fields increase dramatically. Thus, this model is physically consistent.

What subsequently happens to the system? The dust begins to fall toward the center of the body, increasing its mean density and the gravitational radius $r_{g}(r)$ for the mass $M(r)$ at a certain radius $r$. If we would neglect the reciprocal influence of the pressure of the moving matter on the dynamics of the system and on its gravitational field, then after all of the matter has unavoidably fallen and the inequality

$$
r_{g}(r)=2 M(r) \geq r
$$

holds at one of the points $r$ of the system, an event horizon would form at that point according to Schwarzchild's solution for a gravitational field in a vacuum, i.e., the velocity of the falling matter relative to the $r=$ const surfaces would reach the speed of light (see below). Is this what actually happens? The achievement of the speed of light by the matter causes a change in the sign of the interval and is therefore an invariant event, which does not depend on the choice of the reference frame.

An attempt to solve this problem in a reference frame which is stationary at infinity leads at once to a contradiction. In fact, the analytically exact, nonstationary model, in principle, cannot be studied. If, however, a simplification is made and it is assumed that the system is quasistationary at a certain moment in time in the range from one radius to a certain radius known to be large, but still far smaller than the dimensions of the system, then it can be stated, at the very least, that the components $g_{t t}(r)$ and $g_{r r}(t)$ of the metric have singularities (zeros and poles) in this reference frame. [3] |) When the parameters of the system are chosen so that there would be a region in space where the inequality (1) is sure to be satisfied, it becomes clear that the metric does not have singularities, regardless of whether the inequality (1) is satisfied.

This can be shown by assuming that if a singularity appears at a certain point $r_{0}$, the component of the metric near it can be represented in the form

$$
g_{i i}(r) \approx \operatorname{const}\left(r-r_{0}\right)^{y_{i}},
$$

where $y_{i}$ is a certain number. When such a metric is substituted into the equations, it is found that they do not have a solution for any $y_{i} \neq 0$.

This apparently indicates that the singularities and thus the horizon of the rapidly moving matter are eliminated (the right-hand side of the Einstein equations, which is equal to zero in a vacuum, becomes singular in the presence of ultrarelativistic falling matter when the radial component of the three-velocity tends to unity and the radial and temporal components of the four-velocity tend to infinity; this is also the reason for the elimination of the singularities of the metric).

However, in reality all this stems from the inapplicability of the quasistationary approximation in the case of strong gravitational fields. It is inapplicable because the passage of time in the system is highly nonuniform due to the nonuniformity of the component $g_{t t}(r)$ of the metric. This causes the picture, which appears to be stationary far from the center, to become highly nonstationary to an observer approaching the symmetry center of the system.

Nevertheless, this does not remove the question posed: do an horizon and a black hole appear in the real nonstationary case? 
2. An answer to the question posed can be found by selecting a comoving reference frame. The problem was solved in this frame in Ref. 1 (Sec. 103) in the special case of $\alpha=0$ (see below). Matter is at rest in the reference frame chosen, and its motion can be evaluated only from the variation of the "circumferential" or photometric distances $r$, which are related to the center of the system and are defined as the circumferences of the respective circles around the center: $2 \pi r$. When the radius $r$ is defined as such, it is convenient to represent the metric in the form

$$
d s^{2}=e^{\nu} d t^{2}-e^{\lambda} d R^{2}-r^{2}\left(d \theta^{2}+\sin ^{2} \theta d \varphi^{2}\right)
$$

Here $R$ is the coordinate of a dust particle in the comoving reference frame or its index, and $e^{\nu}, e^{\lambda}$, and $r$ are functions of $R$ and $t$. It is noteworthy that at zero pressure, i.e., when $\alpha=0$, we have $\nu=0$, i.e., the reference frame is simultaneously synchronous.

To solve the problem posed we write out the Einstein equation in the comoving reference frame:

$$
\begin{gathered}
r^{\prime 2} e^{-\lambda}\left(1+r \nu^{\prime} / r^{\prime}\right)-e^{-\nu}\left(2 r \ddot{r}+\dot{r}^{2}-r \dot{r} \dot{\nu}\right)=1+8 \pi \alpha r^{2} \varepsilon, \\
2 \dot{\mu}^{\prime}+\dot{\mu} \mu^{\prime}-\dot{\lambda} \mu^{\prime}-\nu^{\prime} \dot{\mu}=0, \\
\left(\lambda+2 \mu+\frac{2}{1+\alpha} \ln \varepsilon\right)=0, \\
\left(\nu+\frac{2 \alpha}{1+\alpha} \ln \varepsilon\right)^{\prime}=0 .
\end{gathered}
$$

Here $\mu=2 \ln r$, a prime denotes differentiation with respect to $R$, and a dot denotes differentiation with respect to $t$. Equations (3) were derived in Ref. [1] [Eqs. (2), (5), and (6) of problem 5 in Sec. 100].

It follows from $(3 \mathrm{~d})$ that

$$
\nu=-\frac{2 \alpha}{1+\alpha} \ln \varepsilon+f^{*}(t)
$$

and that by transforming the time $t$ in the interval element (2) the function $f^{*}(t)$ can be set equal to $[2 \alpha /(1+\alpha)] \ln \varepsilon_{*}$, where $\varepsilon_{*}$ is a constant with the dimensions of energy density, which expresses the measurement scale of $\varepsilon$. Then

$$
\nu=\frac{2 \alpha}{1+\alpha} \ln \frac{\varepsilon}{\varepsilon_{*}} .
$$

We next assign the indices $R$ to the dust particles so that $r=R$ in the initial moment. Under such initial conditions $r^{\prime}(R, t)$ corresponds to $\left(n_{0} / n\right)^{1 / 3}$, where $n(R, t)$ is the concentration of dust particles and $n_{0}$ is its value at the initial moment.

Let us now ascertain the conditions which must be imposed on the initial distribution of the dust. The most important among them is that the inequality (1) need not hold within the matter at the initial moment. It means that there is no horizon in all space in the initial moment. It thus imposes an upper limit on the initial density of the dust and on the initial dimensions of the system. More specifically, if 
the initial density distribution of the dust is set equal to $\varepsilon_{0}(R)$, then, according to (1), the maximum radius of the body $R_{\max }$ is uniquely specified by the expression

$$
R_{\max }=2 \int_{0}^{R_{\max }} 4 \pi \varepsilon_{0}(R) R^{2} d R
$$

Then, it follows from (3c) and (4) that

$$
\frac{\partial}{\partial t}[\alpha(\lambda+2 \mu)-\nu]=0
$$

or

$$
\nu=\alpha\left[\lambda+2 \mu+f^{*}(R)\right],
$$

where $f^{*}(R)$ is an arbitrary function that depends on the initial conditions.

3. Let us now find the initial values for all the variables in our problem. We have already assigned these values for $r$ and $\varepsilon$. From (4) it follows that

$$
\nu_{0}=\frac{2 \alpha}{1+\alpha} \ln \frac{\varepsilon_{0}}{\varepsilon_{*}}
$$

To find the initial value of $\lambda$ we take advantage of the fact that the problem has already been solved for $\alpha=0$, and we can therefore utilize the familiar expression for $\left.\lambda_{0}\right|_{\alpha=0}$ from Ref. [1] (Sec. 103.6):

$$
\lambda_{0}(R)=-\ln [1-S(R)],
$$

where for $\alpha=0$ we have

$$
S(R)=2 M(R) / R
$$

and $M(R)$ is the mass within the radius $R$ at the initial moment.

The expression for $S(R)$ for an arbitrary value of $\alpha$ is the same. It can be obtained from Eq. (4) in problem 5 of Sec. 100 in Ref. [1], where the Einstein equations in matter in the comoving reference frame were found for a centrally symmetric system. We write out this equation:

$$
-e^{-\lambda}\left[\mu^{\prime \prime}+\frac{3}{4} \mu^{\prime 2}-\frac{\mu^{\prime} \lambda^{\prime}}{2}\right]+\frac{1}{r^{2}}+\frac{1}{2 e^{\nu}}\left[\dot{\lambda} \dot{\mu}+(\dot{\mu})^{2} / 2\right]=8 \pi \varepsilon .
$$

Expressing $\mu$ in terms of $r\left(\mu=\ln r^{2}\right)$ and combining similar terms, we can bring this expression into the form

$$
8 \pi r^{\prime} \varepsilon r^{2}=-\left[r\left(r^{\prime 2} e^{-\lambda}-1\right)\right]^{\prime}+\frac{r^{\prime}}{e^{\nu}}\left[\dot{\lambda} r \dot{r}+(\dot{r})^{2}\right] .
$$

Taking into account the expression (8), as well as the fact that, according to the expression (100.23) in Ref. [1], the equality

$$
2 M(r)=\left.\int_{0}^{r} 8 \pi \varepsilon(\tilde{r}, t) \tilde{r}^{2} d \tilde{r}\right|_{t=\text { const }}
$$


holds for the initial moment in time, when $\dot{r}=0$ and $r^{\prime}=1$, we obtain the expression (9) for $S(R)$ after preliminarily integrating (11) over $R$ from 0 to $R$.

Substituting the expression (8) into (6) and taking into account (7), we find that

$$
f^{*}(R)=-\frac{2}{1+\alpha} \ln \frac{\varepsilon_{0}}{\varepsilon_{*}}+\ln [1-S(R)]-\ln R^{4} .
$$

4. Now, plugging (6) into (3b) and dividing everything by $\dot{\mu} \mu^{\prime}$, we obtain the expression

$$
\frac{1}{\dot{r}}\left(2 \ln \mu^{\prime}+\mu-\lambda\right)^{\cdot}=\frac{\nu^{\prime}}{r^{\prime}}=\alpha \frac{\left[\lambda+2 \mu+f^{*}(R)\right]^{\prime}}{r^{\prime}} .
$$

Taking into account that $e^{-\nu}\left(2 r \ddot{r}+\dot{r}^{2}-r \dot{r} \dot{\nu}\right)=\left(e^{-\nu} r \dot{r}^{2}\right) \cdot \dot{r}$ and introducing the notation

$$
U(R, t)=(\dot{r})^{2}, \quad Q(R, T)=r^{\prime 2} e^{-\lambda},
$$

we see that Eq. (3a) can be written as an equation for $U$ :

$$
\frac{\dot{U}}{\dot{r}}+a U=\sigma
$$

where

$$
a(R, t)=\frac{1}{r}\left(1-\frac{r \dot{\nu}}{\dot{r}}\right), \quad \sigma(R, t)=\frac{1}{r}\left[Q\left(1+\frac{r \nu^{\prime}}{r^{\prime}}\right)-1-8 \pi \alpha r^{2} \varepsilon\right] e^{\nu} .
$$

This equation has a solution which satisfies the initial conditions:

$$
U(R, t)=\frac{1}{\gamma^{*}(R, t)} \int_{0}^{t} \gamma^{*}(R, \tilde{t}) \sigma(R, \tilde{t}) \tilde{r} d \tilde{t}, \quad \gamma^{*}(R, t)=\exp \left[\int_{0}^{t} a(R, \tilde{t}) \tilde{r} d \tilde{t}\right] .
$$

Finding $U$, we can obtain an expression for the square of the velocity of the matter relative to the $r=$ const surfaces from the form of the metric (2) (see Appendix 1):

$$
V^{2}(R, t)=U e^{-\nu} e^{\lambda} / r^{2}
$$

The expression for $\gamma^{*}$ can easily be found:

$$
\gamma^{*}(R, t)=C(R) r e^{-\nu}
$$

where the multiplier $C(R)$ for $\gamma^{*}(R, t)$, which does not depend on $t$, can be taken out of the integral sign in (16) and canceled; therefore, it can be set equal to unity. Then

$$
\gamma^{*} \sigma=r^{\prime 2} e^{-\lambda}\left(1+\frac{r \nu^{\prime}}{r^{\prime}}\right)-1-8 \pi \alpha r^{2} \varepsilon .
$$

Alternatively, taking into account that Eq. (13) can now be rewritten as

$$
\frac{\left(2 \ln \mu^{\prime}+\mu-\lambda\right)^{\cdot}}{\dot{r}}=\frac{(\ln Q)^{\cdot}}{\dot{r}}=\frac{\nu^{\prime}}{r^{\prime}},
$$

we obtain

$$
\gamma^{*} \sigma=\frac{(r(Q-1))^{\cdot}}{\dot{r}}-8 \pi \alpha r^{2} \varepsilon
$$


Then (16) is rewritten in the form

$$
U=\frac{e^{\nu}}{r}\left[r(Q-1)-R\left(Q_{0}-1\right)+2 \alpha m(R, t)\right]
$$

where we have introduced the notation

$$
m(R, t)=\int_{t}^{0} 4 \pi \tilde{\varepsilon} \tilde{r}^{2} \tilde{r} d \tilde{t}=\left.\int_{r}^{R} 4 \pi \tilde{\varepsilon} \tilde{r}^{2} \tilde{r} d \tilde{r}\right|_{R=\mathrm{cosnt}} .
$$

5. For $\alpha=0$, taking into account (6), (9), (18), and (20), we can easily obtain an analytically exact expression for $U$ and $V$ :

$$
U_{\alpha=0}=S(R)\left(\frac{R}{r}-1\right)=\frac{2 M(R)}{r}-S(R) .
$$

Substituting this expression into (17), for the velocity we obtain

$$
V_{\alpha=0}^{2}=\frac{2 M(R) / r-S(R)}{1-S(R)} .
$$

Hence $V_{\alpha=0}=1$ when $r=r_{0}=2 M(R)$. This coincides with the results in Sec. 100 of Ref. [1], where the problem has already been solved for this case.

6. Finally, let us consider the location of the horizon 44] in the presence of a nonzero pressure. For this purpose we plug the expressions (20) and (8) for $e^{-\lambda_{0}}$ into formula (17). After some relatively simple transformations, we ultimately obtain

$$
r=\frac{2 M(R)+2 \alpha m(R, t)}{1-Q\left(1-V^{2}\right)} .
$$

As will be shown in Appendix 1, the horizon appears at the point and at the time where the velocity of the falling matter relative to the $r=$ const surfaces reaches unity, i.e., where $V=1$. In addition, the speed of light relative to the falling matter at this site is also, as always, equal to unity.

Hence, according to (24) and (9), the horizon radius $r_{\text {hor }}$ is given by the formula

$$
r_{\text {hor }}=2 M(R)+2 \alpha m\left(R, t_{\text {hor }}\right) .
$$

Thus, the horizon is displaced to a larger radius in comparison to the value in a vacuum $r_{0_{\text {hor }}}=2 M(R)$ by $2 \alpha m\left(R, t_{\text {hor }}\right)$. In this case the quantity $m(R, t)$ has the meaning of the mass which would accumulate if we would join layers of dust with the initial radius $R$ and the thickness $d \tilde{r}(\tilde{t})$ to one another up to the radius $r(R, t)$ at the moment when this $d \tilde{r}$ layer passes through the joining point.

7. Regarding the possible values of $\alpha$ we note that $\alpha=0$ corresponds to dustlike matter without interactions between the particles. The results obtained for them are the same [see (23)] as the results for test particles in a central field of mass $M$ (see Sec. 101 in Ref. [1]). However, of course, such an equation of state of matter cannot correspond to reality near the horizon. It is reasonable to assume that the ultrarelativistic equation of state of matter, in which $\alpha=1 / 3$, holds near 
the horizon. Therefore, the location of the horizon should probably be sought with just such a value of $\alpha$.

8. When $\alpha \neq 0$, it would appear that the falling matter should be slowed under the action of the pressure gradient, and the horizon should therefore form later, i.e., be displaced toward smaller values of $r$, but, as we have just shown, it is displaced toward larger values of $r$ by $2 \alpha m\left(R, t_{\text {hor }}\right)$. What is the reason for this contradiction? It can be seen from the initial equations (3) that the reason should be sought in Eq. (3a). For this purpose we explore Eqs. (3a) and (4) in the initial moment for the case of $\alpha \ll 1$. In that moment $\dot{r}=0$ and $r^{\prime}=1$; therefore, we write

$$
[1-S(R)]\left(1-2 a r \frac{\varepsilon^{\prime}}{\varepsilon}\right)-2 r \tilde{r}\left(\frac{\varepsilon}{\varepsilon_{*}}\right)^{2 \alpha} \approx 1+8 \pi \alpha r^{2} \varepsilon .
$$

Since

$$
\left(\varepsilon / \varepsilon_{*}\right)^{2 \alpha} \approx 1+2 \alpha \ln \frac{\varepsilon}{\varepsilon_{*}}, \quad S(R)=\frac{2 M(R)}{R}, \quad r=R,
$$

then, after performing some relatively simple transformations, in the linear approximation with respect to $\alpha$ we obtain

$$
\tilde{r}=-\frac{G M(R)}{r^{2}}\left[1-2 \alpha \ln \frac{\varepsilon}{\varepsilon_{*}}\right]-\frac{\nabla P}{\rho}\left[1-\frac{2 G M(r)}{r c^{2}}\right]-4 \pi \alpha G r \rho,
$$

where $\rho(R, r)=\varepsilon(R, r) / c^{2}$ is the density of the matter. Here, for the sake of clarity we use the ordinary (Gaussian) system of units with $G \neq 1$ and $c \neq 1$. It can be seen from (26) that the first term corresponds to the ordinary Newtonian force of gravity, and the second term corresponds to the interaction force between the particles, i.e, the pressure gradient (just this force is the cause of the slowing of the fall of the matter in the first stage). The remaining terms do not appear in the equation of motion in the Newtonian approximation (the corrections in square brackets are also neglected in that case), but, as we have already seen, the last term begins to dominate over the second term at high energies; therefore, a shift of the horizon toward larger radii appears. Thus, the contradiction has been resolved. Physically this corresponds to the "gravity of pressure" in the general theory of relativity, which surpasses the gradient terms at high energies.

9. The analysis performed allows us to draw the following conclusions.

First, a shift of the horizon toward a larger radius in comparison to the Schwarzchild radius due to the "gravity of pressure" has been discovered. We stress that this effect is purely dynamic and is not observed in the static case (after all the matter has fallen).

Second, according to the results in Appendix 2, the evolution of the entire system at a constant value of $\alpha$ is completely specified by the energy density distribution profile in the initial moment, i.e., for example, by the normalized density distribution of the matter and by the value of the parameter $S$ at an arbitrary point on this distribution.

If the evolution of only one spherical layer of matter with the index $R$ must be described, it is completely specified by three dimensionless parameters in the initial moment in that layer and, in this sense, does not depend on the initial distribution 
of the matter in the system below and above that layer. However, this in no way signifies the independence of the spherical layers in the general case, since just these three parameters, as will be seen from Appendix 2, govern the interaction of the layers. Consequently, integration of the system leads to a complete family of selfsimilar solutions.

Third, according to Appendix 2, a local extremum appears on the $\left.V(R)\right|_{t=\text { const }}$ curve for a specific choice of initial parameters, and when $V=1$, it leads to the formation of a second apparent horizon in the system (an analog of the second horizon in the Reissner-Nordström and Kerr-Newman solutions for an electrically charged rotating static black hole; for an interpretation of these solutions, see, for example, Refs. [5] and [6]).

\section{1}

We have hitherto used the term horizon to refer to a trapping surface, or an apparent horizon, as it is called in the literature.

Let us ascertain the difference between an event horizon and an apparent horizon in greater detail in an example. We assume that we already have a stationary black hole of mass $M$ and that there is an apparent horizon at $r=2 M$. Now we assume that another chunk of matter with a mass $\delta M$ falls into our black hole. After it falls, the radius of the apparent horizon increases to $2(M+\delta M)$. Thus, if an observer is placed between these radii before the additional chunk of matter falls, he would then be outside the black hole, but after the chunk of matter falls he would be inside it. The concept of an event horizon is global and is determined by the entire course of evolution of the black hole or, stated differently, by all the mass which falls into it at any time.

The existence of an apparent horizon, which specifies a black hole locally, is sufficient for the existence of a black hole. As follows from our arguments, in the spherically symmetric case the two horizons ultimately coincide and form a static black hole described by Schwarzchild's solution. Therefore, we shall henceforth use the term horizon to refer to the apparent horizon.

Let us prove that the horizon in a system with spherical symmetry forms at the moment when a falling particle with a nonzero rest mass achieves the speed of light relative to the $r=$ const surfaces at the same point. For this purpose we write the law of motion for the particle in the form

$$
r(R, T)=R-\int_{0}^{t} \sqrt{U(R, \tilde{t})} d \tilde{t} .
$$

We now assume that we are located on a dust particle with the index $R_{\infty}$ and we are tracking a dust particle with the index $R_{p}$, which sends us a light beam passing through the radii $r_{p}\left(R_{p}, t\right)$, from the large radius $r_{\infty}(t)$. The criterion for determining that the dust particle has not yet reached the horizon is the fact that we still see

light from it, i.e., the light propagating still crosses the radii $r>r_{p}$. Therefore, the criterion for determining that the dust particle has reached the horizon is an event 


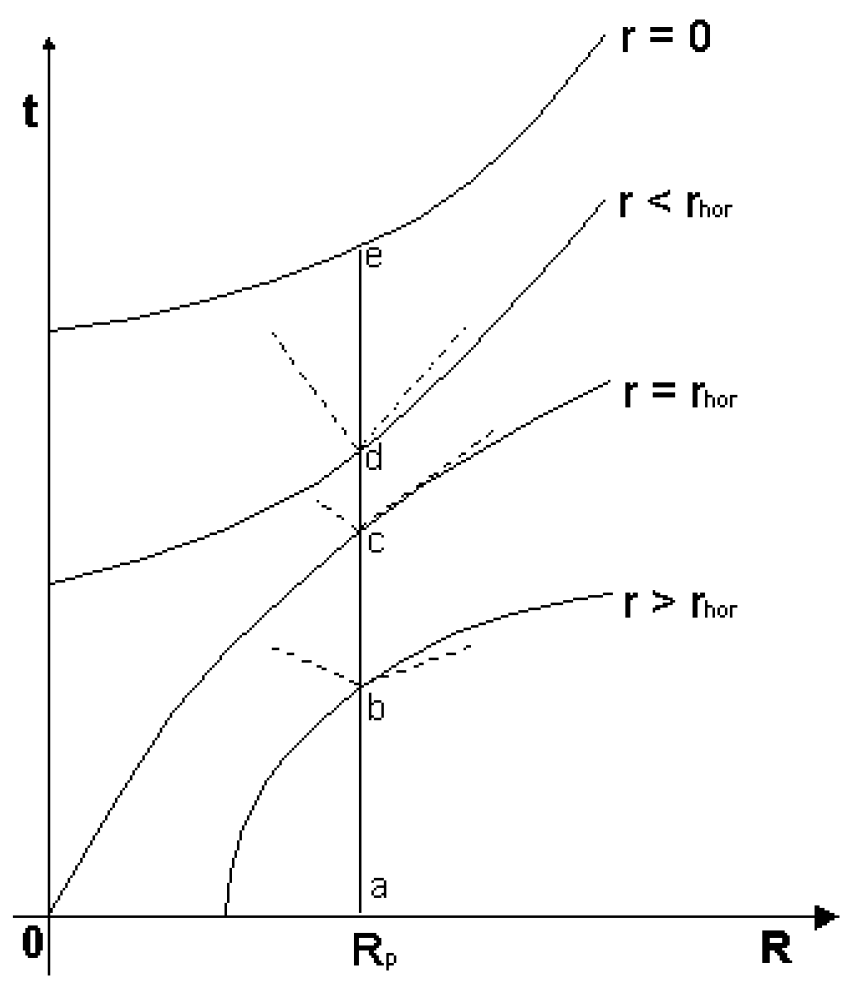

Figure 1:

in which the light propagating from $R_{p}$ can no longer cross the radii $r>r_{p}$. Let us express this criterion mathematically.

In Fig. [ 1 the vertical straight line abcde denotes the world line of an $R_{p}$ dust particle in the coordinates $R$ and $t$ of the comoving reference frame from the moment of rest $(a)$ to the center of the system $(e)$ at $r=0$. In this case of solid curves passing through points $e, d, c$, and $b$ denote, respectively, lines of constant values of $r(R, t)$ for $r=0, r<r_{\text {hor }}, r=r_{\text {hor }}$, and $r>r_{\text {hor }}$. The dashed lines emerging from these points denote the cones within which light emitted by the $R_{p}$ dust particle can propagate (light cannot propagate outside these cones). Therefore, according to the criterion indicated above, the horizon forms at the point where the cone is tangent to the $r=$ const line. In the figure this line is designated as $r=r_{\text {hor }}$, and it passes through point $c$. For clarity, Fig. [ 1 shows that the light cone intersects lines with $r>r_{p}$ at point $b$; therefore, there is still no horizon at that point. This figure also shows that at point $d$ the light cone is located entirely above the $r=$ const curve passing through point $d$. Consequently, this light cone intersects only lines with $r<r_{p}$, and therefore point $d$ is already located below the horizon.

Let us examine the expression (27) on one of the $r=$ const curves and take its complete differential on that curve:

$$
0=d R-\sqrt{U} d t-\frac{1}{2} \int_{0}^{t} \frac{U^{\prime}(R, \tilde{t})}{\sqrt{U(R, \tilde{t})}} d R d \tilde{t},
$$


or

$$
\left.\sqrt{U} \frac{d t}{d R}\right|_{r=\mathrm{const}}=1-\frac{1}{2} \int_{0}^{t} \frac{U^{\prime}(R, \tilde{t})}{\sqrt{U(R, \tilde{t})}} d \tilde{t} .
$$

Next, differentiating (27) with respect to $R$, we obtain the following expression for $r^{\prime}:$

$$
r^{\prime}=1-\frac{1}{2} \int_{0}^{t} \frac{U^{\prime}}{\sqrt{U}} d \tilde{t}
$$

with consideration of which from (28) we find

$$
\left.\frac{d t}{d R}\right|_{r=\mathrm{const}}=\frac{r^{\prime}}{\sqrt{U}} .
$$

Thus, we have found an expression for the slope of an $r=$ const curve relative to the $R$ axis.

To find the slope of a light cone, by definition, for light we have $d s^{2}=0$. Hence, from (2) it follows that

$$
\left.\frac{d t}{d R}\right|_{\text {ligth }}=\sqrt{e^{\lambda-\nu}} .
$$

According to the foregoing statements, the criterion for the absence of a horizon is the condition

$$
\left.\frac{d t}{d R}\right|_{\text {ligth }}<\left.\frac{d t}{d R}\right|_{r=\text { const }} .
$$

Substituting the expressions (29) and (30) therein and taking into account (17), we obtain this criterion in the form

$$
|V|=\frac{\sqrt{U e^{\lambda-\nu}}}{r^{\prime}}<1
$$

Here, according to (29), the rate of motion of the matter relative to the $r=$ const lines has the form

$$
|V|=\left.\frac{d l}{d \tau}\right|_{r=\mathrm{const}}=\left.\sqrt{e^{\lambda-\nu}} \frac{d t}{d R}\right|_{r=\mathrm{const}} .
$$

Thus, the assertion that a horizon forms at the moment when the matter achieves the velocity $V=1$ relative to the $r=$ const surfaces has been proved. The horizon surface separates regions in which $r$ is space-similar and time-similar.

\section{2}

To solve the equations describing collapse, we first bring them into dimensionless forms. For this purpose it is convenient to introduce the following notation:

$$
x=r / R, \quad \gamma=\frac{\rho_{0}(R)}{\langle\rho\rangle}=\frac{8 \pi \varepsilon_{0}(R) R^{2}}{3 S(R)} .
$$


In this Appendix we find the ranges of permissible values of $\gamma$ and $S$, investigate the character of collapse at these values of the parameters, and obtain numerical solutions for $V^{2}$. We must first of all know the form of the function $r^{\prime}(x)$. Differentiating (27), we obtain[5)]'

$$
r^{\prime}(x)=1+\frac{1}{2} R \int_{1}^{x}[\ln U(R, \tilde{x})]^{\prime} d \tilde{x} .
$$

Unfortunately, an analytically exact expression for $r^{\prime}$ can be found only in the case of $\alpha=0$, the character of collapse can be assessed exactly only at that value of $\alpha$. However, the main features of that character, as will be seen below, remain the same as in the case of $\alpha \neq 0$. Therefore, let us first investigate the case of $\alpha=0$.

Thus, we should find $r^{\prime}(R, x)$. According to the expression (22) for $U$, we obtain

$$
\ln [U(R, x)]=\ln [S(R)]+\ln \left(\frac{1}{x}-1\right) .
$$

Introducing the notation $y=r^{\prime}-x$ and taking into account that $x^{\prime}=y / R$, we have

$$
(\ln U)^{\prime}=\frac{S^{\prime}}{S}-\frac{y}{R x(1-x)} .
$$

The substitution of this expression into (33) gives

$$
y(x)+x-1=\frac{1}{2} \int_{1}^{x}\left[\frac{R S^{\prime}}{S}-\frac{y(\tilde{x})}{\tilde{x}(1-\tilde{x})}\right] d \tilde{x} .
$$

Differentiating (34) with respect to $x$, we obtain

$$
\frac{\partial y(x)}{\partial x}+a^{*}(x) y(x)=\sigma^{*}(R)
$$

where we have introduced the notation

$$
a^{*}(x)=\frac{1}{2 x(a-x)}, \quad \sigma^{*}(R)=\frac{R S^{\prime}}{2 S}-1=-\frac{3}{2}+\frac{3}{2} \gamma .
$$

As can be seen, Eq. (35) coincides in form with Eq. (15), and the initial conditions, $\left.y\right|_{t=0}=0$, are the same; therefore, the method used to solve it is similar. The solution has the form

$$
y(x)=r^{\prime}-x=\sigma^{*}\left[\sqrt{\frac{1-x}{x}} \arctan \sqrt{\frac{1-x}{x}}-(1-x)\right] .
$$

Let us find the domain of $r^{\prime}$. First, the condition for compression of the matter has the form $r^{\prime} \leq 1$. Second, the condition that dust layers with different $R$ do not intersect [6) ') has the form $r^{\prime}>0$. Thus,

$$
0<r^{\prime} \leq 1
$$


We assume that the $V^{2}(R)$ curve for $t=t_{m}=$ const has a local extremum, and we presume (to fix ideas) that it is a maximum. Then the horizon appears specifically at the local maximum, i.e., the point $R=R_{\text {extr }}$. We now find the condition for a maximum. First, at that point we should have $V^{2}\left(R_{\text {extr }}, x\right)=V_{\text {extr }}^{2}$. Second, since it is the first point at which the velocity of the matter achieves the value $V_{\text {extr }}$ and the rate of collapse increases with time, in the vicinity of this point we should have $V^{2}<V_{\text {extr }}^{2}$, or

$$
\begin{aligned}
& \frac{\partial V^{2}\left(R, t_{m}\right)}{\partial R}>0, \quad R<R_{\text {extr }}, \\
& \frac{\partial V^{2}\left(R, t_{m}\right)}{\partial R}<0, \quad R>R_{\text {extr }} .
\end{aligned}
$$

If it turns out that (38) holds with opposite inequality signs, there will be a local minimum on the $V^{2}(R)$ curve at the point $R_{\text {extr }}$ at the moment when the velocity $V_{\text {extr }}$ is achieved at that point, i.e., the matter will achieve the velocity $V_{\text {extr }}$ last at that point.

The condition for an extremum is written in the form

$$
\frac{\partial V^{2}(R, x)}{\partial R}=0
$$

where, according to (23),

$$
\left.V^{2}(R, x)\right|_{\alpha=0}=\frac{1-1 / x}{1-a / S(R)}
$$

Differentiating this expression with respect to $R$, we obtain

$$
\left.\frac{\partial V^{2}}{\partial R}\right|_{t=t_{m}}=\frac{S / R}{1-S}\left[-y+\frac{1-3 \gamma}{1-S}\right]
$$

where it has been taken into account that $x^{\prime}=y / R$ and $S^{\prime} / S=(3 \gamma-1) / R$. Then, with allowance for the fact that $0<x \leq 1,0<\gamma \leq 1,-1<y \leq 0$, and $0<S<1$, the condition (38) can be rewritten in the form

$$
\begin{aligned}
& -y>\frac{3 \gamma-1}{1-S}, \quad R<R_{\text {extr }}, \\
& -y<\frac{3 \gamma-1}{1-S}, \quad R>R_{\text {extr }} .
\end{aligned}
$$

If we introduce the notation $z=\sqrt{(1-x) / x}$ and take into account that, according to formula $(23), z=V \sqrt{1 / S-1}$, from (40) we obtain

$$
\frac{3}{2}(1-\gamma)\left[z \arctan (z)-\frac{z^{2}}{1+z^{2}}\right]-\frac{3 \gamma-1}{1-S}>0, \quad R<R_{\mathrm{extr}}
$$




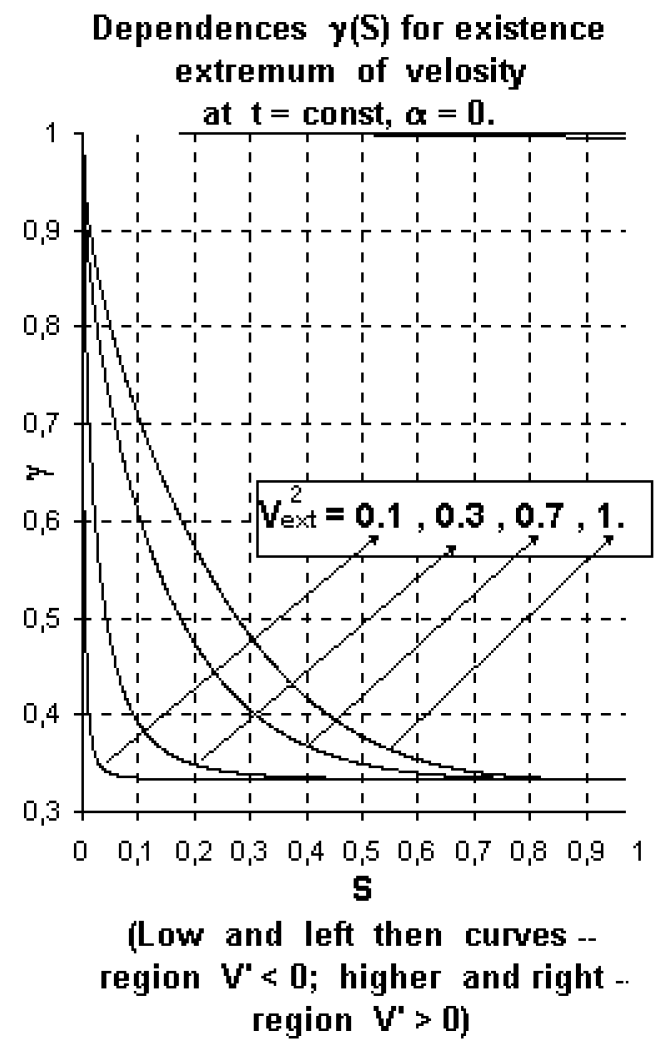

Figure 2:

$$
\frac{3}{2}(1-\gamma)\left[z \arctan (z)-\frac{z^{2}}{1+z^{2}}\right]-\frac{3 \gamma-1}{1-S}<0, \quad R>R_{\text {extr }}
$$

or for the extremum point we can write

$$
(1-\gamma)\left[z \arctan (z)-\frac{z^{2}}{1+z^{2}}+\frac{2}{1-S}\right]-\frac{4 / 3}{1-S}=0 .
$$

This formula can be used to construct the plot of $\gamma\left(S, V_{\text {extr }}\right)$ separating positive and negative values of the derivative $\left(V^{2}\right)^{\prime}$ and to determine the character of the extremum. The corresponding curves for various values of $V_{\text {extr }}$ are shown in Fig. 11. The regions where $V^{\prime}>0$ are located above and to the right of them, and the regions where $V^{\prime}<0$ are located below and to the left of them.

It is seen from Fig. 1 that there can be (for a definite choice of the distribution profile of the matter in the initial moment and of the parameter $S$ at a certain point $R_{*}$ ) two values of $R$, at which $V=1$ at a certain moment in time, and, therefore, the appearance of a second horizon in the system is possible.

The appearance of a second horizon is not news in the physics of black holes (see, for example, the Reissner-Nordström or Kerr-Newman solution in Ref. [7]).

The results obtained in this Appendix apply to the case of the absence of pressure, although the case of $\alpha=1 / 3$ is of experimental interest. Therefore, we used formulas (4), (6), (8), (9), (12), (17), (18), (20), and (33) to introduce new dimensionless 
variables $\left(\hat{\nu}=\nu-\nu_{0}, \hat{\lambda}=\lambda-\lambda_{0}, \hat{U}=U e^{-\nu_{0}}\right.$, and $\left.\hat{\varepsilon}=\varepsilon / \varepsilon_{0}\right)$ and equations for them. The initial conditions for them take the form

$$
\hat{\nu}_{0}=\hat{\lambda}_{0}=\hat{U}_{0}=0, \quad r_{0}^{\prime}=\hat{\varepsilon}_{0}=1
$$

Designating the new coordinates as $x=r / R$ and $\xi=R / R_{*}\left(R_{*}=\right.$ const $)$ and introducing the parameters $[7]$ )

$$
h=\xi \partial_{\xi} \nu_{0}, \quad h_{\mathrm{cr}}=S \frac{1+3 \alpha \gamma}{1-S}, \quad \eta=h / h_{\mathrm{cr}},
$$

we obtain equations for the new variables in the form

$$
\begin{gathered}
e^{\hat{\nu} / \alpha}=x^{4} e^{\hat{\lambda}} \\
\hat{\varepsilon}=\left(e^{-\hat{\nu} / \alpha}\right)^{(1+\alpha) / 2}, \\
\ln \left(r^{\prime 2} e^{-\hat{\lambda}}\right)=\int_{1}^{x}\left[\xi \frac{\partial_{\xi} \hat{\nu}}{r^{\prime}}+\frac{h}{r^{\prime}}\right] d \tilde{x}, \\
\hat{U}=e^{\hat{\nu}}\left[r^{\prime 2} e^{-\hat{\lambda}}(1-S)-1+\frac{S}{x}-\alpha \frac{3 \gamma S}{x} \int_{1}^{x} \hat{\varepsilon} \tilde{x}^{2} d \tilde{x}\right], \\
r^{\prime}=1+\frac{1}{2} \int_{1}^{x} \xi \partial_{\xi}(\ln \hat{U}) d \tilde{x}-\frac{h}{2}(1-x) .
\end{gathered}
$$

In the new variables the velocity is

$$
V^{2}=\frac{\hat{U} e^{\hat{\lambda}-\hat{\nu}}}{\left(r^{\prime}\right)^{2}(1-S)} .
$$

Hence $x_{\text {hor }}=S+2 \alpha \tilde{m}$, where

$$
\tilde{m}=\frac{m}{R}=\varepsilon_{0} R^{2} \int_{x}^{1} 4 \pi \hat{\varepsilon} \tilde{x}^{2} d \tilde{x}, \quad \varepsilon_{0} R^{2}=\frac{3 \gamma S}{8 \pi} .
$$

We note that this formula and formula (36) can be used to find the corrections $\delta r_{\text {hor }}$ in (25) to the displacement of the horizon in the linear approximation with respect to $\alpha$, since, according to Eq. (103.11) from Ref. [1] for $\alpha=0$, we have

$$
8 \pi \varepsilon r^{2}=\frac{2 M^{\prime}}{r^{\prime}}=\frac{8 \pi \varepsilon_{0} R^{2}}{r^{\prime}}
$$

or

$$
\hat{\varepsilon} x^{2}=1 / r^{\prime} .
$$

This expression can be substituted into (43) and a quadrature expression can be obtained for the correction sought. 


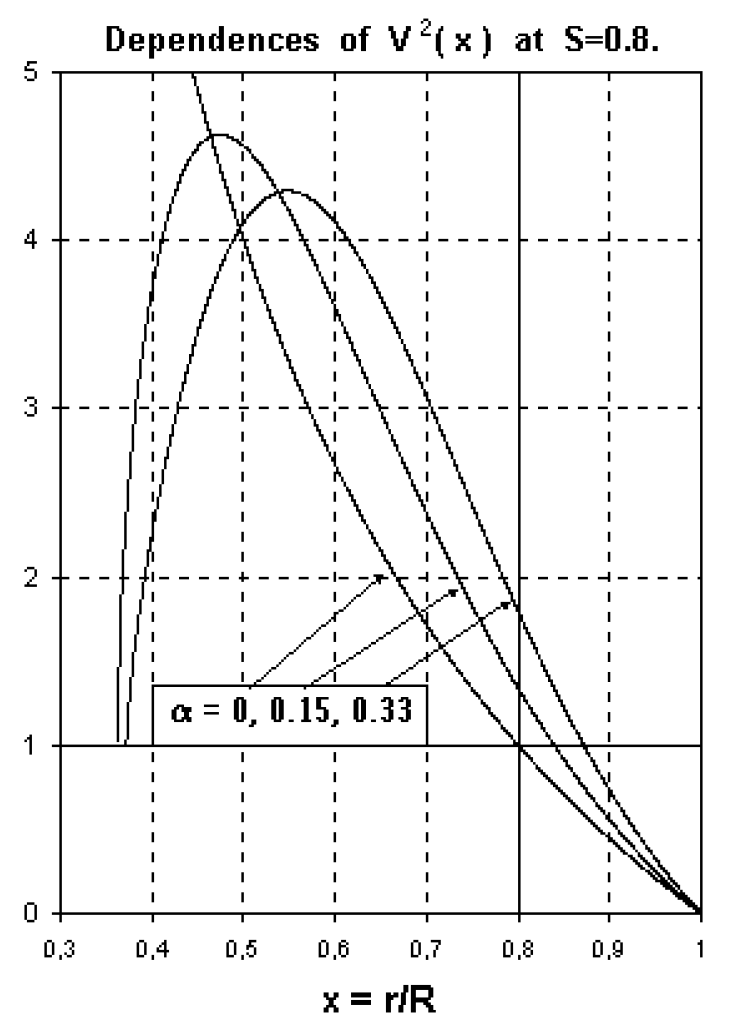

Figure 3:

In addition, we numerically integrated the equations for the case of $\alpha \neq 0$ using a difference scheme, and the results for various values of $\alpha$ are presented in Fig. 1 . As it should be, according to (25), the plots of $V^{2}(x)$ are displaced upward and to the right as $\alpha$ is increased from $\alpha=0$ to $\alpha=1 / 3$.

The numerical calculations confirm that the analytical results of this Appendix remain valid for the real equation of state of matter: $P=\alpha \varepsilon$. Figure 1 shows plots of $\gamma(S), \xi(S)$, and $\eta(S)$ for the special case of a Gaussian density distribution: $\varepsilon_{0}(\xi) / \varepsilon_{0}(0)=\exp \left(-3 \xi^{2}\right)$. Comparing this figure with Fig. 1, we can see that the $\gamma(S)$ curve in Fig. 1 1 crosses the $\gamma(S)$ curves in Fig. 1 in the downward direction roughly at the point $S \approx 0.92$, if we proceed from $\xi=0$ to $\xi=1$. As can be seen in Fig. 1, the point $S \approx 0.92$ corresponds to $\xi \approx 0.85$ and $\eta \approx 0.1$; therefore, since the region where $V^{\prime}>0$ is located above and to the right of the curves in Fig. 1 and the region where $V^{\prime}<0$ is located below and to the left of these curves, the point $\xi \approx 0.85$ should be a local maximum on the $V(\xi)$ curve for a constant value of $t$.

This analytical result is confirmed by a numerical calculation of $\left.V(\xi)\right|_{t=\text { const }}$ curves, whose results are shown in Fig. ?? with the predicted maxima.

To conclude this Appendix we would like to say a few words regarding the initial characteristics and distribution of the matter.

When the equations of the model were brought into dimensionless form, it was found that the solution for a spherical layer of matter with the index $R$ is completely specified by three dimensionless parameters in the initial moment in that layer: $0<S<1,0<\gamma<1$, and $0<\eta<1$. This corresponds to assigning the initial conditions for the gravitational potential and two parameters which deter- 
Dependences of parameters:

$\mathrm{Y}(\mathbf{S}), \eta(\mathbf{S}), \xi(\mathbf{S})$

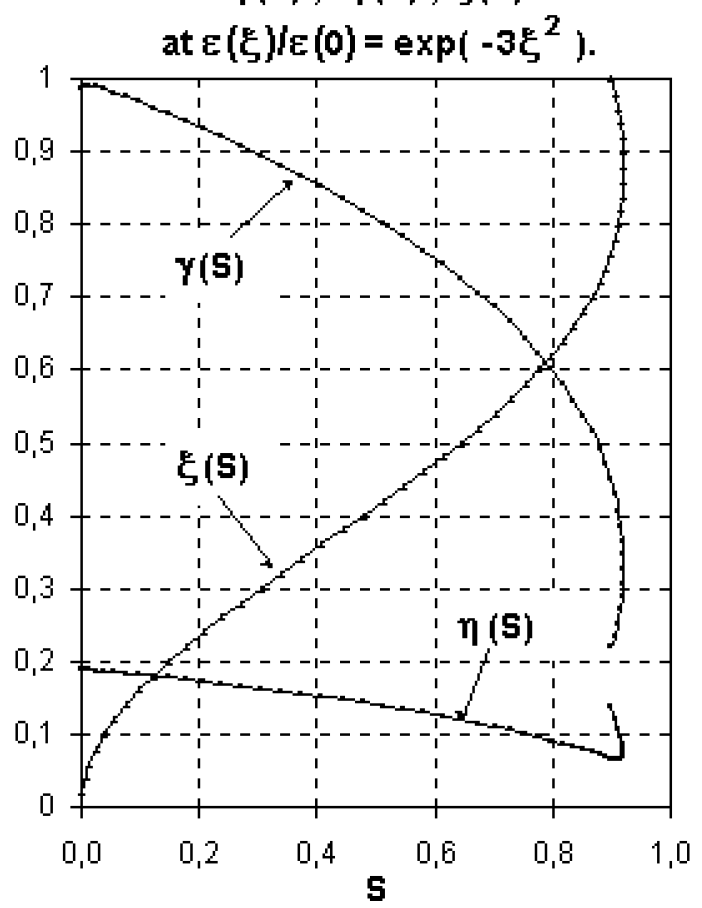

Dependences $V^{2}(\xi)$ at $t=$ const and dependence: $E(\xi)=E(\xi) / E(0)=\exp \left(-3 \xi^{2}\right)$.

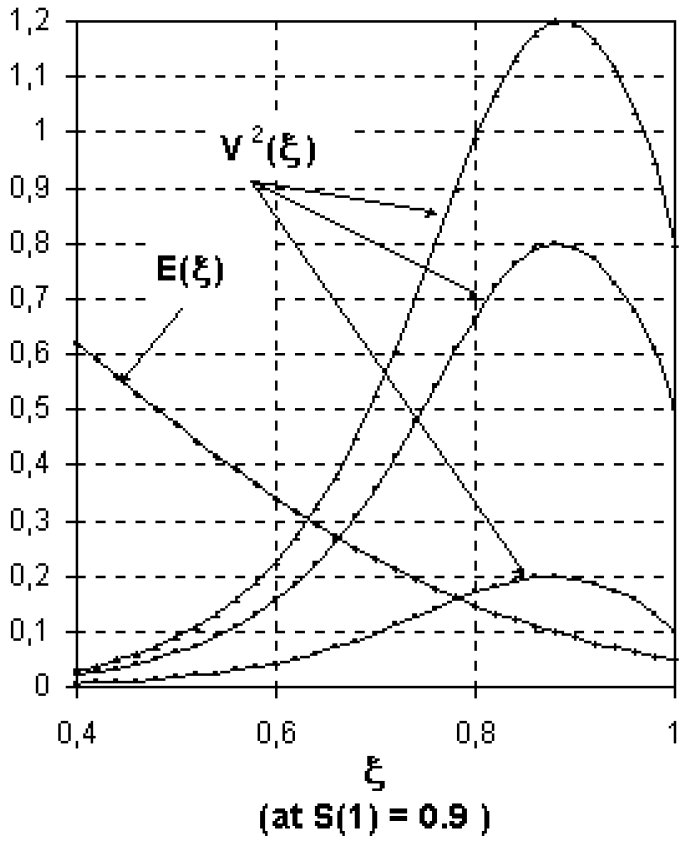

Figure 4:

mine the distribution of the matter and the pressure gradient near the point under consideration. Thus, upon integration we at once find a whole family of self-similar solutions, [8] ) which can be characterized by these three parameters alone and which contains the dependence on the other layers of matter above and below the radius $R$ considered.

We thank N. S. Kardashev, V. L. Ginzburg, B. V. Komberg, V. N. Lukash, and $\mathrm{Yu}$. M. Bruk, as well as all the participants in the seminars of the Division of Theoretical Physics and the Astrocosmic Center of the P. N. Lebedev Physics Institute of the Russian Academy of Sciences for fruitful discussions of this work and for their important comments.

With our sincerest gratitude we recall D. A. Kirzhnits, with whom we formulated the ideas and initial approaches used in the present work.

This work was supported by the Russian Foundation for Basic Research (Grant No. 96-15-96616).

\section{References}

$\left.{ }^{*}\right)$ ] E-mail: aas@srdlan.npi.msu.su

[1)] Only the gravitational forces and the forces of interaction between the dust particles, i.e., the pressure, act on the matter. 
[2)] In case under discussion the word "dust" does not, in any way, imply the absence of interactions between the particles, except in the case of $\alpha=0$.

[3)] When there is an horizon, the components of the metric in the reference frame which is stationary at infinity, like the Schwarzchild metric, must acquire singularities as the surface of that horizon is approached.

[4)] Here we are dealing with the so-called apparent horizon, or, as it is also called, trapping surface, whose definition has been given in Refs. [5] and [6]. As for the event horizon, as will be seen below, after all the matter falls, it coincides with the apparent horizon (for further details, see Appendix 1).

[5)] Here and in the following $d \tilde{x}=\left.(\dot{r} d t / R)\right|_{R=\text { const }}$ and $\partial / \partial x=$ $\left.(R / r)(\partial / \partial t)\right|_{R=\text { const }}$.

[6)] We consider just such a case, since intersection of the layers would signify the appearance of an infinite density already at a radius $r \neq 0$, which is forbidden by cosmic censorship (see, for example, Ref. [6]).

[7)] The significance of $h_{\text {cr }}$ is that, according to (3a), $\ddot{r}=0$ when $h=h_{\text {cr }}$ in the initial moment, i.e., the system is in an unstable equilibrium. Here and in the following $\partial_{\xi}=\partial / \partial \xi$.

[8)] For further information on this subject, see, for example, Ref. [3].

[1] L. D. Landau and E. M. Lifshitz, The Classical Theory of Fields, 4th ed., Pergamon, New York (1975) [Russ. original of newer edition, Nauka, Moscow (1988)].

[2] M. A. Podurets, Dokl. Akad. Nauk SSSR 154, 300 (1964) [Sov. Phys. Dokl. 9, $1(1964)]$.

[3] M. W. Choptuik, Phys. Rev. D 44, 3124 (1991); E-prints archive gr-qc/9607034.

[4] P. S. Wesson, J. Math. Phys. 19, 2283 (1978).

[5] R. Penrose, Structure of Space-Time, W. A. Benjamin, New York (1968) [Russ. transl., Bibfizmat, Mogilev (1972)].

[6] I. D. Novikov and V. P. Frolov, Physics of Black Holes, Kluwer Academic, Dordrecht-Boston (1989) [Russ. original, Nauka, Moscow (1986)].

[7] C. W. Misner, K. S. Thorne, and J. A. Wheeler, Gravitation, W. H. Freeman, San Francisco (1973) [Russ. transl., Mir, Moscow (1977)].

Translated by P. Shelnitz 